\title{
AGRICULTURE ET ENVIRONNEMENT
}

Pendant un certain temps, l'environnement et l'agriculture ont vécu une relation dans laquelle cette dernière pouvait être considérée tour à tour comme victime, et comme source de nuisance.

Victime de pollutions chimiques, de l'urbanisation, de l'emprise de grands travaux, de dégâts de gibier ou même d'espèces protégées. Victime d'être, parfois, le lieu de défoulement de citadins. Les griefs des agriculteurs ne manquent pas et il convient de les examiner. En contrepartie, il faut reconnaître l'existence de nuisances importantes. Par exemple les effets directs ou indirects de l'utilisation des pesticides, ceux de l'utilisation des engrais, les modifications drastiques du milieu, comme le remembrement et le drainage. Bien entendu chacun de ces points doit aussi faire l'objet d'un bilan : comme chaque fois qu'une société se trouve devant une situation conflictuelle, les condamnations sans nuances sont rarement avantageuses. Si on se réfère par exemple au seul problème des engrais azotés, il devient possible de donner aux agriculteurs des conseils d'usage qui maintiennent la rentabilité de la fertilisation tout en abaissant son coût et en réduisant notablement les pertes de nitrates en direction des eaux.

Cependant la relation entre l'agriculture et l'environnement est entrée depuis peu dans une phase nouvelle. On assiste à la reconnaissance de plus en plus marquée du fait que dans un pays façonné par l'homme comme le nôtre, la notion de paysage rural se superpose dans une large mesure à celle de la nature. Et seule l'agriculture peut sans doute gérer cette entité. Les agriculteurs qui ne souhaitent ni abandonner sans espoir de retour des surfaces, ni voir se dégrader leur qualité et craignent l'apparition d'un vide sociologique se rencontrent avec les environnementalistes qui les rejoignent en souhaitant eux garder une diversification des milieux souvent créée par l'agriculture. De nouvelles conditions sont en train de se mettre en place qui obligent les uns et les autres à réfléchir autrement.

L'agriculteur en particulier peut être amené à se pencher sur les avantages que lui apporte le respect de son environnement, même si en apparence cela lui impose des contraintes.

Jacques LECOMTE, Président de la Cellule Environnement, INRA.

\author{
Courrier de la cellule environnement n.2 \\ PROBLEMATIQUES ET DEBATS
}

